\title{
Application value of D-dimer testing and Caprini risk assessment model (RAM) to predict venous thromboembolism (VTE) in Chinese non-oncological urological inpatients: a retrospective study from a tertiary hospital
}

\author{
Zi-Qiang Wu ${ }^{1}$, Kai-Xuan $\mathrm{Li}^{1}$, Quan Zhu ${ }^{1}$, Hao-Zhen $\mathrm{Li}^{1}$, Zheng-Yan Tang ${ }^{1,2}$, Zhao Wang ${ }^{1}$ \\ ${ }^{1}$ Department of Urology, Xiangya Hospital, Central South University, Changsha, China; ${ }^{2}$ Provincial Laboratory for Diagnosis and Treatment of \\ Genitourinary System Disease, Changsha, China \\ Contributions: (I) Conception and design: ZY Tang, Z Wang; (II) Administrative support: ZY Tang; (III) Provision of study materials or patients: ZY \\ Tang, Z Wang; (IV) Collection and assembly of data: ZQ Wu, KX Li, Q Zhu, HZ Li; (V) Data analysis and interpretation: ZQ Wu, KX Li, Q Zhu, \\ HZ Li; (VI) Manuscript writing: All authors; (VII) Final approval of manuscript: All authors. \\ Correspondence to: Zheng-Yan Tang; Zhao Wang. Department of Urology, Xiangya Hospital, Central South University, Changsha, China. \\ Email: xytzyan@163.com; xywangz07@163.com.
}

\begin{abstract}
Background: The Caprini risk assessment model (RAM) and D-dimer testing have been widely used in the prediction of venous thromboembolism (VTE). However, the clinical significance of these testing options are limited in non-oncological urological inpatients because of the low specificity.

Methods: This retrospective study included 1,453 patients who were admitted to the non-oncological unit of the Department of Urology, Xiangya Hospital, Central South University, from January 2018 to December 2018. The highest score of Caprini RAM and the highest D-dimer level were collected in this retrospective study. Ultrasound examinations of the lower extremities or computed tomographic pulmonary arteriography (CTPA) were applied to patients who were suspected of having VTE, if necessary.

Results: A total of 1,453 patients were collected in this study, which included 34 VTE and 1,419 non-VTE patients. The threshold of D-dimer was $0.89 \mu \mathrm{g} / \mathrm{mL}$, according to the receiver operating characteristic (ROC) curve, with a sensitivity of $82.4 \%$, a specificity of $83.3 \%$, and a negative predictive value (NPV) of $99.5 \%$. The cut-off of the Caprini RAM was 5, with a sensitivity of $76.5 \%$, a specificity of $58.7 \%$, and an NPV of 99.0\%. The area under the curve (AUC) was higher for D-dimer (0.86) than for the Caprini score (0.73).

Conclusions: The application of $0.89 \mu \mathrm{g} / \mathrm{mL}$ and a score of 5 as cut-offs for D-dimer testing and Caprini RAM, respectively, could safely decrease the proportion of Chinese non-oncological urological inpatients who needed to undergo further examinations. These new findings may enhance the application value of D-dimer testing and Caprini RAM for Chinese non-oncological urological inpatients.
\end{abstract}

Keywords: Venous thromboembolism (VTE); Caprini risk assessment model (Caprini RAM); D-dimer testing; urology; non-oncological inpatients

Submitted Jan 10, 2020. Accepted for publication Jul 20, 2020.

doi: $10.21037 /$ tau-20-320

View this article at: http://dx.doi.org/10.21037/tau-20-320

\section{Introduction}

Venous thromboembolism (VTE), which consists primarily of deep vein thrombosis (DVT) and pulmonary embolism $(\mathrm{PE})$, is a common and severe complication leading to considerable morbidity and mortality in urological inpatients (1). The risk of death in patients diagnosed with VTE is 13.5 times higher than those without VTE (2). Moreover, the clinical signs and symptoms of VTE are 
not specific to detect VTE in advance (3). Thus, it is of great importance to take adequate measures to predict and prevent VTE.

The Caprini risk assessment model (RAM) have been recommended for evaluating the risk of VTE for nonorthopedic surgical patients (4). A recent research has shown that Caprini RAM based on individual risk factors, has a better predictive ability than Padua RAM in the evaluation of VTE (5). However, the validity of Caprini RAM should be questioned since a high proportion of non-oncological urological patients would be classified as moderate-risk or high-risk. D-dimer testing has also been used to predict the risk of VTE since it has been observed that D-dimer level is usually elevated in patients with VTE. Both sensitivity and negative predictive value (NPV) of D-dimer testing are high when using $0.5 \mu \mathrm{g} / \mathrm{mL}$ as the cut-off value (6). However, using a low $\mathrm{D}$-dimer threshold is associated with poor specificity. This situation has limited the widespread clinical application of D-dimer testing (7). Moreover, recent studies have shown that the D-dimer level would significantly rise after urologic oncology surgery (8). To date, few studies that concerning the clinical significance of the D-dimer level and Caprini RAM have been conducted to predict VTE in non-oncological urological inpatients.

The purpose of this study was to investigate the appropriate threshold of D-dimer and Caprini RAM by retrospectively evaluating the predictive value of Caprini RAM and D-dimer in Chinese non-oncological urological inpatients.

We present the following article in accordance with the TRIPOD reporting checklist (available at http://dx.doi. org/10.21037/tau-20-320).

\section{Methods}

\section{Patients}

This study reviewed the data of all inpatients who were admitted to the non-oncological unit of the Department of Urology, Xiangya Hospital, Central South University, from January, 2018, to December, 2018. No physical prophylaxis or drug prophylaxis was routinely used if the patient was not diagnosed with VTE before surgery. DVT was diagnosed by lower extremities ultrasounds, and PE was diagnosed by computed tomographic pulmonary arteriography (CTPA) when appropriate. Caprini score was evaluated for every hospitalized patient before surgery and 3 consecutive days after surgery. The highest score of Caprini RAM was selected before VTE. After admission, the D-dimer examination was routinely performed pre-operatively and post-operatively for all patients, and the highest D-dimer value was selected before VTE. Ultrasound for lower extremities was performed among patients if their D-dimer level or Caprini score indicated a risk of VTE according to VTE guidelines in Xiangya Hospital. On the other hand, ultrasound for lower extremities or CTPA would be prescribed to rule out VTE when some symptoms of those prone to suspect VTE were present. We consulted the VTE group in Xiangya Hospital for treatment options, as well as follow-up regimen if the patient was diagnosed with VTE by ultrasonography or CTPA. Ethical approval was given by the ethics committee of Xiangya Hospital, Central South University (2019030078). The study complies with the Declaration of Helsinki (as revised in 2013). This was a retrospective study; informed consent was not necessarily required. The retrospective data were collected through an electronic medical recording system through patients' ID numbers. The exclusion criteria of the study were as follows: (I) age under 18 years old, (II) surgery to remove urological cancer during the current admission, (III) postoperative pathological examination revealing malignancies, (IV) incomplete record. The baseline demographics and clinical data of these patients were consistent with the previous study we had submitted to another journal. (Wang Z, 2020, unpublished data).

\section{Caprini RAM}

The Caprini RAM was used to evaluate VTE in this study. This rating scale included a total of 39 risk factors. The individual scores of each risk factor were summed to generate a cumulative risk score that defined the patient's VTE risk level: low risk 1-2, moderate risk $3-4$ and high risk $\geq 5$.

\section{D-dimer measurement}

D-dimer levels were measured in the Clinical Laboratory Center of Xiangya Hospital. Fresh plasma was used for these analyses. The results were expressed in $\mu \mathrm{g} / \mathrm{mL}$ (fibrinogen equivalent units). According to the test results, patients with D-dimer level less than $0.5 \mu \mathrm{g} / \mathrm{mL}$ was categorized as non-VTE group. Otherwise, the patient was advised to be screened for the $\mathrm{D}$-dimer again or undergo 
Table 1 Comparison of D-dimer $(\mu \mathrm{g} / \mathrm{mL})$ and Caprini score in VTE group and non-VTE group

\begin{tabular}{lcc}
\hline Variate & VTE & Non-VTE \\
\hline $\mathrm{N}$ & 34 & 1419 \\
$\mathrm{D}-$ dimer $(\mu \mathrm{g} / \mathrm{mL})$ & $6.90 \pm 9.65$ & $0.73 \pm 2.17$ \\
Caprini score & $5.76 \pm 1.96$ & $4.35 \pm 1.47$ \\
\hline
\end{tabular}

The D-dimer $(\mu \mathrm{g} / \mathrm{mL})$ and Caprini score were expressed as mean \pm standard deviation. VTE, venous thromboembolism.

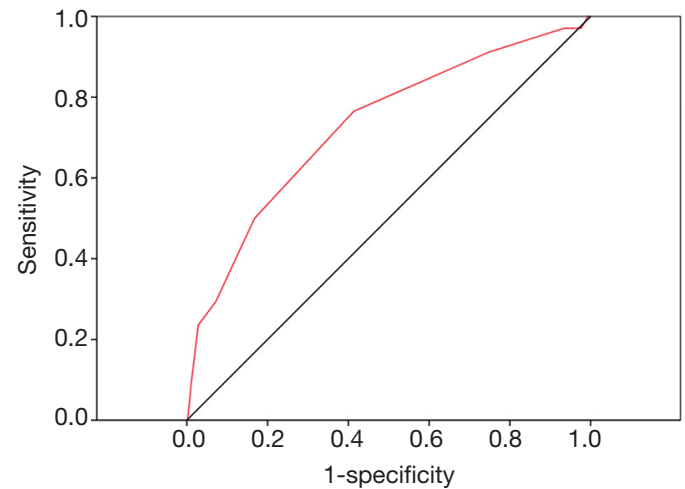

Figure 1 ROC curve for Caprini RAM to discriminate VTE and non-VTE patients. ROC, receiver operating characteristic; RAM, risk assessment model; VTE, venous thromboembolism.

ultrasonography for further diagnosis if necessary.

\section{Statistical analysis}

Statistical analysis was performed by the SAS version 9.3 software (SAS Institute Inc, Cary, NC, USA). Quantitative data were expressed as the mean \pm standard deviation and analyzed by the independent $t$-test. Qualitative data were expressed as a percentage (\%) and analyzed by the $\chi^{2}$ test. A $\mathrm{P}$ value of $<0.05$ was considered to be statistically significant. The receiver operating characteristic (ROC) curve was plotted by referring to the sensitivity $v s$. 1-specificity of the D-dimer level and Caprini score. The areas under the curve (AUCs) and cut-off values were also calculated. Those higher than the cut-off value were positive, and those lower than the cut-off values were negative. Sensitivity, specificity, NPV, and positive predictive value (PPV) were used to estimate the value of these two methods based on the cut-off value. In addition, the Youden index, was used to evaluate the accuracy of the Caprini RAM and D-dimer testing in this study.

\section{Results}

A total of 1,453 non-oncological urological inpatients were enrolled in our study, which included 34 VTE and 1,419 non-VTE patients from January, 2018, to December, 2018, in the non-oncological units of the urologic department. In a previous study submitted to another journal (under review), we suggested that $\mathrm{D}$-dimer and Caprini score were two risk factors for VTE. When we compared the groups of VTE and non-VTE, significant differences were observed in the $\mathrm{D}$-dimer level $(\mathrm{P}=0.001$; Table 1) and Caprini score $(\mathrm{P}=0.000 ;$ Table 1).

We conducted a ROC curve analysis to evaluate the ability of the Caprini score to discriminate VTE and nonVTE patients (Figure 1). Based on the ROC results, the AUC value of Caprini RAM was 0.73 (95\% CI: 0.64-0.82; $\mathrm{P}=0.000$ ), and the cut-off value was 5 . In other words, when the threshold of the Caprini score was 5, the sum of sensitivity and specificity was the highest for discriminating VTE and non-VTE patients. A total of 841 patients had a Caprini score of less than 5, with the majority (833/841, $99 \%)$ of these patients being in the non-VTE group; meanwhile, 612 patients had a Caprini score above or equal to 5 , with a small minority $(26 / 612,4.2 \%)$ being in the VTE group (Table 2).

We further conducted ROC analysis to value the diagnostic power of the D-dimer in distinguishing VTE from non-VTE (Figure 2). Based on the statistical results, the AUC value of $\mathrm{D}$-dimer was 0.86 (95\% CI: $0.78-0.94 ; \mathrm{P}=0.000$ ), and the cut-off value was $0.89 \mu \mathrm{g} / \mathrm{mL}$. This result means that when the threshold of D-dimer was $0.89 \mu \mathrm{g} / \mathrm{mL}$, the sum of sensitivity and specificity was the highest for discriminating VTE and non-VTE patients. In total, 1,188 patients had a D-dimer level less than $0.89 \mu \mathrm{g} / \mathrm{mL}$, and the majority $(1,182 / 1,188,99.5 \%)$ of these patients were in the non-VTE group; meanwhile 265 patients had a D-dimer level above or equal to $0.89 \mu \mathrm{g} / \mathrm{mL}$, and a small minority $(28 / 265,10.6 \%)$ of these patients were in the VTE group (Table 3). 
Table 2 Use 5 as the threshold of Caprini score to discriminate VTE and non-VTE patients

\begin{tabular}{lccc}
\hline Caprini score & VTE group & non-VTE group & Total \\
\hline$\geq 5$ & $26(76.5 \%)$ & $586(41.3 \%)$ & 612 \\
$<5$ & $8(23.5 \%)$ & $833(58.7 \%)$ & 841 \\
Total & 34 & 1,419 & 1,453 \\
\hline
\end{tabular}

VTE, venous thromboembolism.

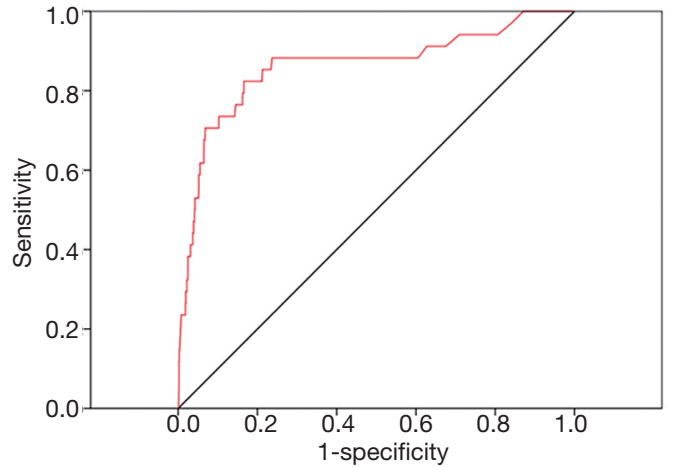

Figure 2 ROC curve for D-dimer to discriminate V'TE and nonVTE patients. ROC, receiver operating characteristic; VTE, venous thromboembolism.

The sensitivity, specificity, NPV, and PPV were described in Table 4 when threshold 5 and $0.89 \mu \mathrm{g} / \mathrm{mL}$ were correspondingly applied to Caprini RAM and D-dimer test. The ROC curve analysis was used to compare application the value of $\mathrm{D}$-dimer with Caprini RAM. As shown in Figure 3, the AUC of the D-dimer was significantly higher than that of the Caprini RAM (AUC: $862 \pm 0.04$ vs. $0.73 \pm 0.05$; $95 \%$ CI: $0.78-0.94$ vs. 0.64-0.82), which indicated a higher forecast accuracy of the $\mathrm{D}$-dimer. Also, the Youden index was 0.66 for D-dimer when the critical point was $0.89 \mu \mathrm{g} / \mathrm{mL}$. However, the Youden index in the Caprini RAM was only 0.35 when the critical point was set at 5 .

\section{Discussion}

This study was designed to investigate the appropriate threshold of D-dimer and Caprini RAM and to compare the predictive values of these two methods in Chinese non-oncological urological patients in a tertiary hospital. D-dimer, which is one of the most reliable and accurate biochemical indicator, is widely used in the clinical diagnosis of VTE and is the most promising indicator that has been studied thus far. It has been recommended for the prediction of VTE by the guidelines of the American College of Chest Physicians (4). However, D-dimer testing is known to have high sensitivity but low specificity detection. These limitations have reduced its clinical application for predicting VTE, and the value of D-dimer for predicting thrombosis is controversial (9-11). Furthermore, few studies have investigated the appropriate threshold of D-dimer in non-oncological urological inpatients.

D-dimer, as a fibrin-related marker, usually increases in the process of thrombosis while several other factors, such as surgery (12), aging, malignancy, cardiovascular diseases, and renal dysfunction, may also be associated with an elevation of D-dimer $(13,14)$. In our study, the D-dimer level of VTE patients was significantly higher than that of non-VTE patients $(6.90 \pm 9.65$ vs. $0.73 \pm 2.17$; $\mathrm{P}=0.001)$. This result was consistent with previous studies $(8,15)$. Traditionally, VTE would consider by clinicians if the $\mathrm{D}$-dimer level is over $0.5 \mu \mathrm{g} / \mathrm{mL}(16,17)$. However, in the non-oncological urological department, the collected data showed a high proportion of inpatients with a D-dimer level higher than $0.5 \mu \mathrm{g} / \mathrm{mL}$. ROC curve analysis of D-dimer demonstrated an AUC of 0.86 , a sensitivity of $82.4 \%$, a specificity of $83.3 \%$, and an NPV of $99.5 \%$ when the appropriate threshold of D-dimer was set at $0.89 \mu \mathrm{g} / \mathrm{mL}$. In other words, the appropriate threshold of D-dimer in nononcological urological patients was elevated to $0.89 \mu \mathrm{g} / \mathrm{mL}$. These findings are consistent with researches conducted by other authors. For instance, Shi et al. reported that the appropriate threshold of D-dimer rose to $0.98 \mu \mathrm{g} / \mathrm{mL}$, with a sensitivity of $83.9 \%$ and a specificity of $80.0 \%$ in urological tumor surgery inpatients (8). Additionally, Shi et al. revealed that the appropriate threshold of D-dimer rose to $1.5 \mu \mathrm{g} / \mathrm{mL}$ with a sensitivity of $87.5 \%$, a specificity of $93.8 \%$, and an NPV of $99.2 \%$ in gynecologic malignancy inpatients (18). Finally, a recent review found that the D-dimer was gradually increased with aging (19). To promote the appropriate application of $\mathrm{D}$-dimer testing in a clinical setting, several strategies have been proposed, such 
Table 3 Use $0.89 \mu \mathrm{g} / \mathrm{mL}$ as the threshold of D-dimer to discriminate VTE and non-VTE patients

\begin{tabular}{lccc}
\hline D-dimer $(\mu \mathrm{g} / \mathrm{mL})$ & VTE group & non-VTE group & Total \\
\hline$\geq 0.89$ & $28(82.4 \%)$ & $237(16.7 \%)$ & 265 \\
$<0.89$ & $6(17.6 \%)$ & $1,182(83.3 \%)$ & 1,188 \\
Total & 34 & 1,419 & 1,453 \\
\hline
\end{tabular}

VTE, venous thromboembolism.

Table 4 Sensitivity, specificity, NPV, and PPV of Caprini RAM and D-dimer when the threshold was set at 5 and $0.89 \mu \mathrm{g} / \mathrm{mL}$, respectively

\begin{tabular}{lcccc}
\hline Variate & Sensitivity (\%) & Specificity (\%) & NPV (\%) & PPV (\%) \\
\hline Caprini score & 76.5 & 58.7 & 99.0 & 4.2 \\
D-dimer & 82.4 & 83.3 & 99.5 & 10.6 \\
\hline
\end{tabular}

NPV, negative predictive value; PPV, positive predictive value; RAM, risk assessment model.

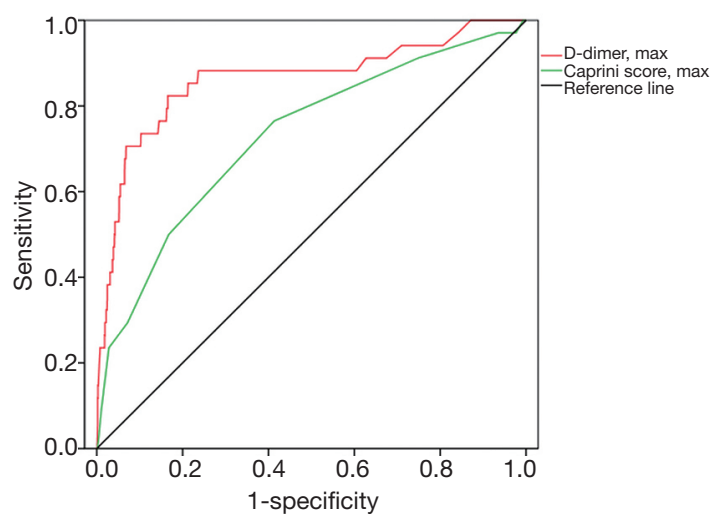

Figure 3 ROC curve for D-dimer and Caprini RAM to discriminate VTE and non-VTE patients. ROC, receiver operating characteristic; RAM, risk assessment model; VTE, venous thromboembolism.

as using age-adjusted D-dimer interpretation and clinical probability-adjusted interpretation. Both of these options have increased the clinical application of D-dimer testing by raising the $\mathrm{D}$-dimer threshold to categorize results as positive $(20,21)$. This is somewhat different from the traditional threshold of $0.5 \mu \mathrm{g} / \mathrm{mL}$. We speculate that these differences arise from the fact that the D-dimer threshold traditionally set at a low level to maximize sensitivity and NPV. In addition, these differences might have also been caused by the heterogeneity.

There are a number of assessment schemes for evaluating VTE. These include Caprini (22), Kucher (23), Padua (24), and Wells (25), among many others. Two RAMs are recommended to predict VTE in the ninth edition of the clinical practice guidelines developed by the American College of Chest Physicians (26). Caprini RAM is suggested for non-orthopedic surgical patients, While Padua RAM is recommended in internal medicine patients for VTE risk assessment. These assessments are also recommended by the Chinese expert consensus on the prevention of venous thrombosis (4). Furthermore, several clinical studies in China have demonstrated that Caprini RAM is more applicable for Chinese patients than other risk assessments $(5,27)$.

All the patients enrolled in our study were evaluated for the risk of VTE by the Caprini RAM. The Caprini score of VTE patients was significantly higher than that of non-VTE patients $(5.76 \pm 1.96$ vs. $4.35 \pm 1.47 ; \mathrm{P}=0.000)$. These results are consistent with previous studies $(28,29)$. However, the stratified system of Caprini RAM seems unable to sufficiently discriminate VTE in non-oncological urological inpatients since most inpatients would be classified as moderate-risk or high-risk, a fact which has also been observed in other studies. Chen et al. reported that Caprini score 4 could be considered as the suitable DVT cut-off level for Chinese internal medicine and surgical patients (5). Shi et al. reported that a Caprini score over 7 may a be better predictor of the occurrence of VTE for malignant tumor patients (18). In our study, ROC analysis demonstrated that the AUC of Caprini score was 0.73, and the appropriate threshold was 5 . The threshold had a sensitivity of $76.5 \%$, a specificity of $58.7 \%$, and an NPV of $99.0 \%$. This discrepancy might be due to the fact that Caprini RAM was designed for a Western population, and 
the variety of diseases we studied was differed from those studies in Western countries. Furthermore, compared to large-scale studies, the number of cases was limited in our study.

A Caprini score being discordant with the levels of D-dimer may lead to an incorrect or confused VTE diagnosis, and thus the relationship between the two methods in predicting VTE requires further clarification. Based on the ROC analysis, the AUC of the D-dimer was significantly higher than that of the Caprini RAM (AUC: $0.86 \pm 0.04$ vs. $0.73 \pm 0.05$; $95 \%$ CI: $0.78-0.94$ vs. $0.64-0.82$ ), which indicates a higher prediction accuracy of the $\mathrm{D}$-dimer.

There are several limitations of our study that should be addressed. First, our study was a retrospective study, and we did not conduct a prospective sample size calculation. In this respect, our study was merely exploratory in nature. Second, although many types of diseases were collected in our study, the number of patients suffering from each disease was small. Third, the cases collected in our study were ranged widely in age, and we cannot rule out age as a potential confounding factor. Finally, due to the limitation of the experimental conditions, we only investigated the inpatients while outpatients were not included in the study. Therefore, further researches on the risk factors of VTE for different diseases and different ages in non-oncological urological patients is needed.

\section{Conclusions}

The appropriate threshold of D-dimer in Chinese nononcological urological inpatients rose to $0.89 \mu \mathrm{g} / \mathrm{mL}$. The risk score of the Caprini RAM above 5 was found to be the optimal VTE cut-off for Chinese non-oncological urological inpatient in this single-center study. The D-dimer level, an objective laboratory indicator, had more application value than the Caprini RAM in predicting VTE among hospitalized non-oncological urological patients. However, further verifications on the $\mathrm{D}$-dimer testing by multicenter studies are still required.

\section{Acknowledgment}

We thank to Qin Jiabi, a professor from the Xiangya School of Public Health, Central South University, for the assistance on statistical analysis.

Funding: None.

\section{Footnote}

Reporting Checklist: The authors have completed the TRIPOD reporting checklist. Available at http://dx.doi. org/10.21037/tau-20-320

Data Sharing Statement: Available at http://dx.doi. org/10.21037/tau-20-320

Conflicts of Interest: All authors have completed the ICMJE uniform disclosure form (available at http://dx.doi. org/10.21037/tau-20-320). The authors have no conflicts of interest to declare.

Ethical Statement: The authors are accountable for all aspects of the work in ensuring that questions related to the accuracy or integrity of any part of the work are appropriately investigated and resolved. Ethical approval was given by the ethics committee of Xiangya Hospital, Central South University (2019030078). The study complies with the Declaration of Helsinki (as revised in 2013). This was a retrospective study; informed consent was not necessarily required.

Open Access Statement: This is an Open Access article distributed in accordance with the Creative Commons Attribution-NonCommercial-NoDerivs 4.0 International License (CC BY-NC-ND 4.0), which permits the noncommercial replication and distribution of the article with the strict proviso that no changes or edits are made and the original work is properly cited (including links to both the formal publication through the relevant DOI and the license). See: https://creativecommons.org/licenses/by-nc-nd/4.0/.

\section{References}

1. Streiff MB, Agnelli G, Connors JM, et al. Guidance for the treatment of deep vein thrombosis and pulmonary embolism. J Thromb Thrombolysis 2016;41:32-67.

2. Alberts BD, Woldu SL, Weinberg AC, et al. Venous thromboembolism after major urologic oncology surgery: a focus on the incidence and timing of thromboembolic events after 27,455 operations. Urology 2014;84:799-806.

3. Huisman MV, Klok FA. Diagnostic management of acute deep vein thrombosis and pulmonary embolism. J Thromb Haemost 2013;11:412-22.

4. Gould MK, Garcia DA, Wren SM, et al. Prevention of 
VTE in nonorthopedic surgical patients: Antithrombotic Therapy and Prevention of Thrombosis, 9th ed: American College of Chest Physicians Evidence-Based Clinical Practice Guidelines. Chest 2012;141:e227S-77S.

5. Chen X, Pan L, Deng H, et al. Risk assessment in Chinese hospitalized patients comparing the Padua and Caprini scoring algorithms. Clin Appl Thromb Hemost 2018;24:127S-35S.

6. Legnani C, Cini M, Scarvelis D, et al. Multicenter evaluation of a new quantitative highly sensitive $\mathrm{D}$-dimer assay, the Hemosil D-dimer HS 500, in patients with clinically suspected venous thromboembolism. Thromb Res 2010;125:398-401.

7. Takach Lapner S, Julian JA, Linkins LA, et al. Comparison of clinical probability-adjusted D-dimer and ageadjusted D-dimer interpretation to exclude venous thromboembolism. Thromb Haemost 2017;117:1937-43.

8. Shi A, Huang J, Wang X, et al. Postoperative D-dimer predicts venous thromboembolism in patients undergoing urologic tumor surgery. Urol Oncol 2018;36:307.e15-21.

9. Hamidi S, Riazi M. Cutoff values of plasma d-dimer level in patients with diagnosis of the venous thromboembolism after elective spinal surgery. Asian Spine J 2015;9:232-8.

10. Hasegawa $M$, Wada $H$, Yamaguchi $T$, et al. The evaluation of D-dimer levels for the comparison of fibrinogen and fibrin units using different D-dimer kits to diagnose VTE. Clin Appl Thromb Hemost 2018;24:655-62.

11. Mohmad Sallih N, Subbiah I, Ali A, et al. Reference ranges for D-dimer levels in Malaysian women in the three trimesters of pregnancy. Malays J Pathol 2019;41:7-13.

12. Kabrhel C, Mark Courtney D, Camargo CA Jr, et al. Factors associated with positive D-dimer results in patients evaluated for pulmonary embolism. Acad Emerg Med 2010;17:589-97.

13. Righini M, Van Es J, Den Exter PL, et al. Age-adjusted D-dimer cutoff levels to rule out pulmonary embolism: the ADJUST-PE study. JAMA 2014;311:1117-24.

14. Bates SM. D-dimer assays in diagnosis and management of thrombotic and bleeding disorders. Semin Thromb Hemost 2012;38:673-82.

15. Sugimoto E, Kuroda T, Fujita Y, et al. D-dimer testing cannot rule out thromboembolism after major lower extremity arthroplasties and thromboprophylaxis treatment. J Anesth 2015;29:686-9.

16. Han C, Zhao Y, Cheng W, et al. The performance of age-adjusted $\mathrm{D}$-dimer cut-off in Chinese outpatients with suspected venous thromboembolism. Thromb Res 2015;136:739-43.
17. Tran HA, Gibbs H, Merriman E, et al. New guidelines from the Thrombosis and Haemostasis Society of Australia and New Zealand for the diagnosis and management of venous thromboembolism. Med J Aust 2019;210:227-35.

18. Shi J, Ye J, Zhuang X, et al. Application value of Caprini risk assessment model and elevated tumor-specific D-dimer level in predicting postoperative venous thromboembolism for patients undergoing surgery of gynecologic malignancies. J Obstet Gynaecol Res 2019;45:657-64.

19. Schouten HJ, Geersing GJ, Koek HL, et al. Diagnostic accuracy of conventional or age adjusted D-dimer cutoff values in older patients with suspected venous thromboembolism: systematic review and meta-analysis. BMJ 2013;346:£2492.

20. Douma RA, le Gal G, Sohne M, et al. Potential of an age adjusted D-dimer cut-off value to improve the exclusion of pulmonary embolism in older patients: a retrospective analysis of three large cohorts. BMJ 2010;340:c1475.

21. van Es J, Beenen LF, Douma RA, et al. A simple decision rule including $\mathrm{D}$-dimer to reduce the need for computed tomography scanning in patients with suspected pulmonary embolism. J Thromb Haemost 2015;13:1428-35.

22. Caprini JA, Arcelus JI, Reyna JJ. Effective risk stratification of surgical and nonsurgical patients for venous thromboembolic disease. Semin Hematol 2001;38:12-9.

23. Kucher N, Koo S, Quiroz R, et al. Electronic alerts to prevent venous thromboembolism among hospitalized patients. N Engl J Med 2005;352:969-77.

24. Barbar S, Noventa F, Rossetto V, et al. A risk assessment model for the identification of hospitalized medical patients at risk for venous thromboembolism: the Padua Prediction Score. J Thromb Haemost 2010;8:2450-7.

25. Wells PS, Anderson DR, Rodger M, et al. Derivation of a simple clinical model to categorize patients probability of pulmonary embolism: increasing the models utility with the SimpliRED D-dimer. Thromb Haemost 2000;83:416-20.

26. Kahn SR, Lim W, Dunn AS, et al. Prevention of VTE in nonsurgical patients: Antithrombotic Therapy and Prevention of Thrombosis, 9th ed: American College of Chest Physicians Evidence-Based Clinical Practice Guidelines. Chest 2012;141:e195S-226S.

27. Liu X, Liu C, Chen X, et al. Comparison between Caprini and Padua risk assessment models for hospitalized medical patients at risk for venous thromboembolism: a retrospective study. Interact Cardiovasc Thorac Surg 2016;23:538-43. 
28. Luo XY, Zhang FX. Validation of the Caprini risk assessment model for venous thromboembolism in Chinese hospitalized patients in a general hospital. Zhonghua Yi Xue Za Zhi 2017;97:1875-7.

Cite this article as: $\mathrm{Wu} Z \mathrm{ZQ}, \mathrm{Li} \mathrm{KX}$, Zhu Q, Li HZ, Tang ZY, Wang $Z$. Application value of $\mathrm{D}$-dimer testing and Caprini risk assessment model (RAM) to predict venous thromboembolism (VTE) in Chinese non-oncological urological inpatients: a retrospective study from a tertiary hospital. Transl Androl Urol 2020;9(5):1904-1911. doi:10.21037/tau-20-320
29. Luksameearunothai K, Sa-Ngasoongsong P, Kulachote $\mathrm{N}$, et al. Usefulness of clinical predictors for preoperative screening of deep vein thrombosis in hip fractures. BMC Musculoskelet Disord 2017;18:208. 